\title{
Asociación entre el dolor anterior postquirúrgico y la reproducción inadecuada de la línea articular postquirúrgica en artroplastia primaria de rodilla
}

\author{
Association between post-surgical anterior pain and inadequate \\ reproduction of the post-surgical joint line in primary knee arthroplasty
}

Claudia Karina Pérez García, * Carlos Eduardo Antonio Romero, ${ }^{\ddagger}$ Luis Alberto De Santiago Esquivel, $\$$ Angélica Marisela Carrillo Sánchez, ${ }^{\S}$ Ricardo Cortés Cerdađ

Citar como: Pérez GCK, Antonio RCE, De Santiago ELA, Carrillo SAM, Cortés CR. Asociación entre el dolor anterior postquirúrgico y la reproducción inadecuada de la línea articular postquirúrgica en artroplastia primaria de rodilla. Acta Med. 2021; 19 (1): 57-60. https://dx.doi.org/10.35366/98571

\section{Resumen}

Introducción: La reproducción de la línea articular es uno de los indicativos más importantes para determinar los resultados satisfactorios de la cirugía protésica de rodilla, pero son pocos los estudios enfocados a la revisión de estos casos a mediano plazo, y menos referente al desarrollo de dolor crónico. Material y métodos: Estudio retrospectivo, longitudinal y observacional, se incluyeron los casos de personas sometidas a una artroplastia total de rodilla primaria en un periodo que abarca de 2017 a 2019 y que contaran con un seguimiento de un año postquirúrgico. Resultados: De los 32 casos tras un año de valoración, sólo 14 (44\%) reportaron presencia de dolor crónico. El análisis de asociación entre dolor y presencia de desplazamiento o restauración en la línea articular mostró ser significativo $(p=0.0001)$, con el test exacto de Fisher. Conclusiones: La línea articular es un factor importante para determinar el rango de movimiento de rodilla, la ausencia de su restauración se relaciona con dolor anterior postquirúrgico a largo plazo.

Palabras clave: Dolor anterior crónico, artroplastia de rodilla, línea articular postquirúrgica.

\section{Abstract}

Introduction: Reproduction of the articular line is one of the most important indexes to determine the satisfactory results of prosthetic knee surgery but there are few studies focused on the review of these cases in a medium term and less, in the development of chronic pain. Material and methods: Retrospective, longitudinal and observational study; which includes patients with a total primary knee arthroplasty in a period of 2017 to 2019 and whom will have a follow-up of a post-surgical year. Results: Of the 32 cases after one year of evaluation only $14(44 \%)$ cases reported the presence of chronic pain. The analysis of the association between pain and the presence of displacement or restoration in the joint line showed to be significant $(p=0.0001)$, with Fisher's exact test. Conclusions: Although the articular line is an important factor in determining the flexion, extension and range of movement, we conclude that the no restoration of the articular line is related to the development of the anterior post-surgical pain in a long term.

Keywords: Chronic anterior pain, knee replacement, postsurgical joint line.

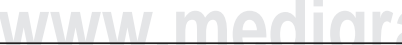

* Médico Residente de cuarto año de Ortopedia y Traumatología, Hospital Regional "Gral. Ignacio Zaragoza", ISSSTE. Facultad Mexicana de Medicina de la Universidad La Salle. Ciudad de México, México.

* Especialidad de Traumatología y Ortopedia, Hospital Regional “Gral. Ignacio Zaragoza", ISSSTE. Ciudad de México, México.

$\S$ Médico Residente de tercer año de Traumatología y Ortopedia, Hospital Regional "Gral. Ignacio Zaragoza", ISSSTE. Ciudad de México, México.
"Especialidad de Traumatología y Ortopedia, Hospital Regional $1^{\circ}$ de Octubre, ISSSTE. Ciudad de México, México.

Correspondencia:

Claudia Karina Pérez García

Correo electrónico: dra.claudiatyo@gmail.com

Aceptado: 22-05-2020.

www.medigraphic.com/actamedica

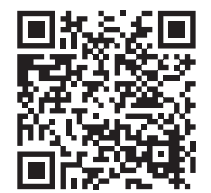




\section{INTRODUCCIÓN}

La artroplastia total de rodilla es un tratamiento disponible para el tratamiento de la osteoartritis sintomática que ha sido refractaria a los tratamientos conservadores y que presentan cambios que afectan múltiples compartimientos de la articulación. ${ }^{1}$ Su uso se ha incrementado en los últimos años, a tal grado que se espera que para el 2030 se realicen 3.48 millones de procedimientos de artroplastia total de rodilla anualmente en todo el mundo. ${ }^{2}$

Ahora bien, los resultados son influenciados por diversos factores como: el peso del paciente, edad, tipo de rehabilitación, genética entre otros. ${ }^{3,4}$ Otro de los factores importantes es como tal el confrontar la pérdida del hueso y la insuficiencia de tejido blando, lo cual genera el reto de la restauración de las dimensiones articulares previas a la cirugía. ${ }^{5}$

Sin embargo, un factor sumamente importante para la recuperación de los pacientes con artroplastia es la recuperación de la línea articular, ya que el restablecimiento de la misma es indispensable para recuperar un adecuado rango de movimiento, estabilidad de los ligamentos colaterales, mejor apoyo para la superficie de carga y duración de la prótesis. ${ }^{6}$ Por lo que una línea articular incorrecta se ha asociado directamente con la inestabilidad articular y el desarrollo de dolor anterior de rodilla.?

Ninguno de los métodos radiográficos disponibles actualmente para medir el nivel de la línea articular (LA) son comparables y por tanto los resultados y la definición de una línea articular baja o alta deben estar relacionados con cada método. ${ }^{8}$ Sin embargo, se ha demostrado que el tubérculo abductor es una referencia que puede emplearse con mayor fiabilidad para medir la LA, ya que es relativa- mente sencillo determinar radiográficamente el cambio en la curvatura a nivel de la metáfisis femoral. ${ }^{6}$

\section{MATERIAL Y MÉTODOS}

Se realizó un estudio retrospectivo, longitudinal y observacional, no involucró experimentación o intervención alguna en los pacientes, ya que en todos los casos se extrajo la información del expediente clínico.

Muestra de pacientes. En el presente estudio se incluyeron a los pacientes que fueron sometidos a un procedimiento de artroplastia total de rodilla primaria en el Hospital Regional "Gral. Ignacio Zaragoza" del ISSSTE en el periodo comprendido entre enero de 2017 y enero de 2019, los cuales además debieron mantenerse en un seguimiento postquirúrgico de al menos un año.

Métodos de evaluación. Se consideró para la evaluación la radiografía previa al procedimiento quirúrgico y la que se realizó para la evaluación de los resultados después de la cirugía. En ambos casos se valoró la proyección anteroposterior. La línea articular se evaluó tomando como referencia el tubérculo abductor en la proyección anteroposterior (Figura 1A-B). Se consideró como un resultado normal aquel en que la línea articular postoperatoria se encontró entre $+4 \mathrm{~mm}$ (desplazamiento hacia distal de la línea articular) y $-4 \mathrm{~mm}$ (desplazamiento hacia proximal de la línea articular). Este resultado es obtenido a partir de la resta entre la línea postoperatoria menos la preoperatoria, tomando como valor 0 aquel en el cual la distancia se encontraba al mismo nivel. ${ }^{5}$

Análisis estadísticos. El análisis estadístico se llevó a cabo empleando el programa IBM SPSS v. 25 (USA). Para
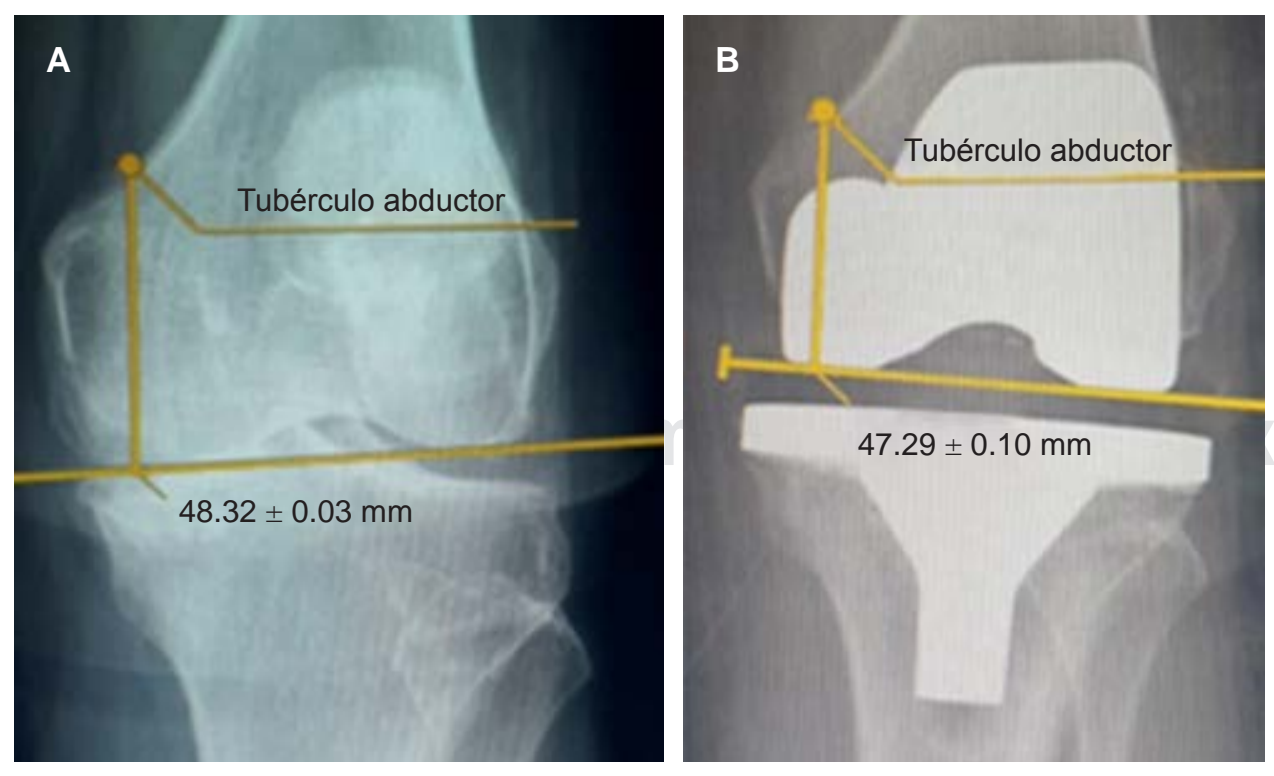

Figura 1:

A) Determinación de la línea articular previo a la cirugía en la proyección anteroposterior. B) Determinación de la línea articular postquirúrgica de la artroplastia total de rodilla primaria en la proyección anteroposterior. Demuestran reproducción adecuada de la línea articular. 
la valoración de variables cualitativas se empleó análisis de frecuencias, mientras que para variables cuantitativas se realizó el cálculo de la media, desviación estándar y rango. Para la asociación se decidió utilizar la prueba exacta de Fisher por tratarse de una muestra pequeña y de variables dicotómicas. Se consideró como valor significativo una $p$ $\leq 0.05$.

\section{RESULTADOS}

Se registraron 32 casos que cumplieran con los criterios de selección en el periodo de evaluación, 18 (56\%) casos correspondieron al lado derecho. Predominó el género masculino con 18 casos (56\%). La edad promedio de la población estudiada fue de una media y DE de $64.94 \pm 7.66$.

Con relación a la medición de la línea articular previo a la artroplastia total de rodilla la media y DE fue de $5.44 \pm 1.347 \mathrm{~mm}$, con un rango entre 3.5-8.5 mm. Mientras que en la valoración de la línea articular al año de la cirugía mostró una media y DE de $5.59 \pm 1.43 \mathrm{~mm}$ y rango de 3.5-7.9. Sin observar diferencia significativa entre la media prequirúrgica y postquirúrgica $(\mathrm{t}=$ 0.9424, $\mathrm{p}=0.3609$ ).

La variación de la línea articular previa a la cirugía y posterior a la misma tuvo promedio y DE de $1.55 \pm 5.07 \mathrm{~mm}$, presentando rango de variación muy amplia. Sin embargo, tomando en cuenta la restauración de la línea articular según el método descrito por Hofmann A y colaboradores, se encontraron 12 pacientes con reproducción adecuada de la línea articular (37.5\%); 12 pacientes con desplazamiento hacia distal (37.5\%) y ocho con desplazamiento a proximal (25.0\%) (Tabla 1).

De los 32 casos tras un año de valoración, sólo 14 (44\%) reportaron presencia de dolor crónico. Después se realizó un análisis de asociación entre el dolor y si se presentó un aumento o disminución en la línea articular, 10 presentaron desplazamiento a distal y cuatro desplazamiento a proximal, realizando un análisis de asociación entre el dolor y si se presentó desplazamiento o restauración en la línea articular, con lo cual se ubtuvo un resultado significativo con el test exacto de Fisher $p=0.0001$. Es importante mencionar que de los 12 casos que presentaron reproducción adecuada de la línea articular ninguno presentó dolor anterior crónico de rodilla (Tabla 2).

\section{DISCUSIÓN}

Como se ha reportado en diversos estudios, la edad promedio de los pacientes sometidos a una artroplastia total es por arriba de los 60 años, lo cual concuerda con lo que se observó en nuestro estudio. 9,10 Respecto a la prevalencia de género en los casos sometidos a este tipo de procedimiento, nuestros resultados son similares a los que se han reportado en poblaciones atendidas en el ISSSTE y el IMSS. 6,11

Es interesante que el resultado en relación al género que se ha reportado en nuestro país difiere con otras latitudes, ya que en todos los países se presenta mayor incidencia en mujeres. ${ }^{12,13}$

Un estudio previo valoró si una diferencia mayor o menor de $8 \mathrm{~mm}$ entre la línea articular prequirúrgica y postquirúrgica se asoció con aumento de la subluxación de rótula, así como la mejora de amplitud de movimiento. ${ }^{5}$ Sin embargo, se desconocía si la variación o ausencia de ésta se asocia con dolor, de igual manera no se tenía información si había una relación directa entre la variación milimétrica de la línea articular y el desarrollo de dolor anterior a largo plazo.

Vera-Avilés ${ }^{6}$ y colaboradores demostraron en un estudio previo que cuando la línea articular se excede de los $4 \mathrm{~mm}$, existe un déficit de la extensión completa, que se refleja como una contractura en flexión, afectando la marcha y el apoyo adecuado, así como la distribución de cargas en zonas poco estables, lo que lleva al aflojamiento temprano de la prótesis; ocurre lo contrario cuando la línea articular es de menos de $4 \mathrm{~mm}^{6}$ sin embargo, no mencionan la relación de la no restauración de la línea articular con el dolor anterior postquirúrgico.

Es más frecuente el desplazamiento hacia distal de la línea articular postquirúrgica que el desplazamiento a proximal, a diferencia de Gómez Vallejo ${ }^{14}$ y su equipo, quienes afirman que el desplazamiento a proximal se relaciona con

\begin{tabular}{|c|c|c|c|c|}
\hline & $\mathrm{n}$ & $\%$ & \% válido & $\%$ acumulado \\
\hline Desplazamiento distal & 12 & 37.5 & 37.5 & 37.5 \\
\hline Restaurada & 12 & 37.5 & 37.5 & 75.0 \\
\hline Desplazamiento proximal & 8 & 25.0 & 25.0 & 100.0 \\
\hline Total & 32 & 100.0 & 100.0 & \\
\hline
\end{tabular}


Tabla 2: Relación entre la línea articular postquirúrgica y dolor anterior crónico.

\begin{tabular}{|c|c|c|c|c|c|}
\hline & & \multicolumn{3}{|c|}{ Línea articular } & \multirow[b]{2}{*}{ Total } \\
\hline \multicolumn{2}{|c|}{ Dolor anterior crónico } & $\begin{array}{c}\text { Desplazamiento } \\
\text { distal }\end{array}$ & Restaurada & $\begin{array}{c}\text { Desplazamiento } \\
\text { proximal }\end{array}$ & \\
\hline \multirow[t]{2}{*}{ Sí } & Recuento & 10 & 0 & 4 & 14 \\
\hline & \% dentro de línea articular & 83.3 & 0.0 & 50.0 & 43.8 \\
\hline \multirow[t]{2}{*}{ No } & Recuento & 2 & 12 & 4 & 18 \\
\hline & \% dentro de línea articular & 16.7 & 100.0 & 50.0 & 56.3 \\
\hline \multirow[t]{2}{*}{ Total } & Recuento & 12 & 12 & 8 & 32 \\
\hline & \% dentro de línea articular & 100.0 & 100.0 & 100.0 & 100.0 \\
\hline
\end{tabular}

rótula baja, sobrecarga y pinzamiento, originando dolor anterior de rodilla. Se observa que en el desplazamiento a distal es más frecuente la presencia de dolor anterior postquirúrgico.

\section{CONCLUSIONES}

Se conoce que la línea articular es un factor importante para determinar el rango de movimiento, por lo que concluimos que la no restauración de la línea articular postquirúrgica en artroplastia primaria de rodilla se relaciona con el desarrollo de dolor anterior postquirúrgico a largo plazo, independientemente de si la línea articular aumenta o disminuye, mientras se mantenga dentro del margen de $\pm 4 \mathrm{~mm}$, considerando de esta manera la restauración de ésta.

Por lo anterior, debe ser una preocupación para el cirujano la restauración de la línea articular postquirúrgica, mediante una correcta planeación prequirúrgica y la toma de decisiones al momento de la artroplastia total de rodilla. Ya que más de la mitad de los pacientes de este estudio presentan desplazamiento de la línea articular.

\section{REFERENCIAS}

1. Varacallo MA, Herzog L, Toossi N, Johanson NA. Ten-year trends and independent risk factors for unplanned readmission following elective total joint arthroplasty at a large urban academic hospital. J Arthroplasty. 2017; 32 (6): 1739-1746.

2. Kurtz S, Ong K, Lau E, Mowat F, Halpern M. Projections of primary and revision hip and knee arthroplasty in the United States from 2005 to 2030. J Bone Joint Surg Am. 2007; 89 (4): 780-785.

3. Chaudhry H, Ponnusamy K, Somerville L, McCalden RW, Marsh J, Vasarhelyi EM. Revision rates and functional outcomes among severely, morbidly, and super-obese patients following primary total knee arthroplasty: a systematic review and meta-analysis. JBJS Rev. 2019; 7 (7): e9.

4. Henderson KG, Wallis JA, Snowdon DA. Active physiotherapy interventions following total knee arthroplasty in the hospital and inpatient rehabilitation settings: a systematic review and metaanalysis. Physiotherapy. 2018; 104 (1): 25-35.

5. Ranera M, Gómez Vallejo J, Seral García B, Seral Iñigo F. La importancia de la línea articular en la revisión de la artroplastia total de rodilla. Rev Esp de Cir Osteoartic. 2009; 44 (237): 15-21.

6. Vera-Avilés FA, Negrete-Corona J, Jiménez-Aquino JM. Artroplastia total de rodilla, pronóstico al restablecer la línea articular. Acta Ortop Mex. 2012; 26 (6): 362-368.

7. Figgie HE 3rd, Goldberg VM, Heiple KG, Moller HS 3rd, Gordon NH. The influence of tibial-patellofemoral location on function of the knee in patients with the posterior stabilized condylar knee prosthesis. J Bone Joint Surg Am. 1986; 68 (7): 1035-1040.

8. Hernandez-Trillo PM. Influencia del nivel de la línea articular sobre el resultado funcional de prótesis de rodilla realizadas con técnica navegada [Tesis]. Madrid: Universidad Complutense de Madrid; 2018.

9. Kulshrestha V, Datta B, Mittal G, Kumar S. Epidemiology of revision total knee arthroplasty: a single center's experience. Indian J Orthop. 2019; 53 (2): 282-288.

10. Durán CJ, Crispin ND. Artroplastia total de rodilla: evaluación funcional y complicaciones. Traumatología y Ortopedia-Hospital Obrero No 1, 2010-2015. Cuad Hosp Clin. 2018; 59: 27-33.

11. Salvatori-Rubí J, Montiel-Jarquín AJ, López-Cázares G, BarragánHervella R, Ortíz-Arellano R, García-Carrasco M et al. Prótesis total de rodilla por gonartrosis grado IV. Acta Ortop Mex. 2014; 28 (3): 193-196.

12. Malik AT, Mufarrih SH, Ali A, Noordin S. Predictors of an increased length of stay following Total Knee Arthroplasty-Survey Report. J Pak Med Assoc. 2019; 69 (8): 1159-1163.

13. Carvalho RT, Lopes TL, Takano MI, Lima JHS, Arrebola LS, Colombo ML et al. Evolution and projection of knee arthroplasties from 2003 to 2030 in the state of São Paulo. Rev Assoc Med Bras (1992). 2019; 65 (7): 1001-1006.

14. Gómez VJ, Albareda AJ, Seral GB, Blanco RN, Ranera GM, Ezquerra HL. La importancia de la restauración de la línea articular en la revisión de la artroplastia total de rodilla. Índices funcionales, calidad de vida y supervivencia. Acta Ortop Mex. 2017; 31 (5): 222-227. 